Case Report

\title{
Surgical aspects using an exoscope for corpus callosotomy: initial experience for epilepsy surgery
}

\author{
Kyoichi Tomoto ${ }^{1}$, Toshiki Nozaki $^{2}$, Chikanori Inenaga ${ }^{1}$, Ayataka Fujimoto ${ }^{1,2, *}$ \\ ${ }^{1}$ Department of Neurosurgery, Seirei Hamamatsu Ceneral Hospital, Hamamatsu, 430-8558 Shizuka, Japan \\ ${ }^{2}$ Comprehensive Epilepsy Center, Seirei Hamamatsu Ceneral Hospital, Hamamatsu, 430-8558 Shizuka, Japan \\ *Correspondence: afujimotoscienceacademy@gmail.com (Ayataka Fujimoto)
}

\section{DOI:10.31083/j.jin2101039}

This is an open access article under the CC BY 4.0 license (https://creativecommons.org/licenses/by/4.0/). Submitted: 15 March 2021 Revised: 25 March 2021 Accepted: 8 May 2021 Published: 28 January 2022

The exoscope is a new form of optic device that has begun to be used in the last decade. The exoscope provides high-quality magnification and illumination at depth, allowing easy manipulation of surgical instruments even in narrow surgical fields. Exoscopic corpus callosotomy (CC) has not been reported. We report herein our initial experience with the utility of exoscopic CC. A 3-year-old boy diagnosed with West syndrome at 4 months old was referred to our hospital. As the seizure spasms were considered to carry a risk of severe traumatic injury, we performed exoscopic CC as palliative therapy. An EX VITOM $3 \mathrm{D}$ exoscope and $4 \mathrm{~K}-3 \mathrm{D}$ surgical monitor were used during the procedure. No surgical complications that could be related to exoscope use were noted. Operative time for exoscopic CC was comparable to that for microscopic CC, despite a lack of previous experience with the equipment. Stereoscopic vision was easily obtained. The exoscope was perceived as providing higher quality magnification. We report our first clinical experience of exoscopic CC. The exoscope appears useful in the field of epilepsy surgery.

\section{Keywords}

Exoscope; Three-dimensional; Corpus callosotomy; Epilepsy surgery; Microscope

\section{Introduction}

The exoscope is a new kind of optic device that has started to see use in the last decade. Since Mamelak et al. [1] first reported their experience using an exoscope in surgeries in 2008, exoscope use has been reported in otolaryngology, spine surgery, hand surgery, plastic surgery and a number of other surgical fields [2-5]. Similarly, exoscope use has recently been reported in the field of neurosurgery, especially brain tumor surgery and cerebrovascular surgery [6-8]. As an exoscope provides high-quality magnification and illumination at depth, operators are able to manipulate the surgical instruments easily even in a narrow surgical field. Moreover, the exoscope offers a three-dimensional (3D) perception of the surgical field using multiple cameras and parallax images, giving the surgeon more comfortable operability.

Corpus callosotomy (CC) has traditionally been recognized as a microsurgical technique, as with other neurosurgical procedures. In contrast, little experience with endoscopic callosotomy has been reported [9-11]. We have started to recognize the utility of an endoscopy that can resolve some of the difficulties encountered in microsurgery to disconnect the rostrum and genu of the corpus callosum. We thus thought the exoscope could offer a surgical alternative for CC without facing the difficulties of disconnecting the rostrum and genu of the corpus callosum entailed in microscopic callosotomy. Exoscopic CC has not previously been reported. To confirm the utility of exoscopic CC, we performed exoscopic CC for a pediatric patient with medically intractable epilepsy.

\section{Materials and methods}

Submission of this case report was approved by the ethics review board at Seirei General Hospital, and written informed consent was obtained from the parents of the patient for the exoscopic CC procedure and publication of the case details.

A 3-year-old boy who had been diagnosed with West syndrome at 4 months old was referred to our hospital. He exhibited epileptic spasms with eye deviation to the right side, tonic seizures, and loss of consciousness consistent with atypical absence. The frequency of epileptic spasms was approximately 10 times/day. Seizures were intractable to multiple anticonvulsants and physical and intellectual development had ceased. Magnetic resonance imaging showed an atrophic brain for his age, particularly for the bilateral frontotemporal lobes. Multiple deep sulci surrounded by thickened gray matter were evident. Long-term video electroencephalography (EEG) showed generalized slow spike-waves, and paroxysmal generalized fast activities during sleep. However, since the EEG showed occipital-dominant 5- to $8-\mathrm{Hz}$ theta and alpha background activities that were incompatible with the findings of hypsarrhythmia, we regarded his condition as a phase of predisposition to Lennox-Gastaut syndrome. Ictal EEG of spasms showed an electrodecremental pattern with or without subsequent tonic EEG patterns.

As no epileptic focality had been detected, we suggested that the patient undergo CC or vagus nerve stimulation (VNS) therapy. The patient initially underwent VNS therapy. Although the frequency of tonic seizures was reduced, 


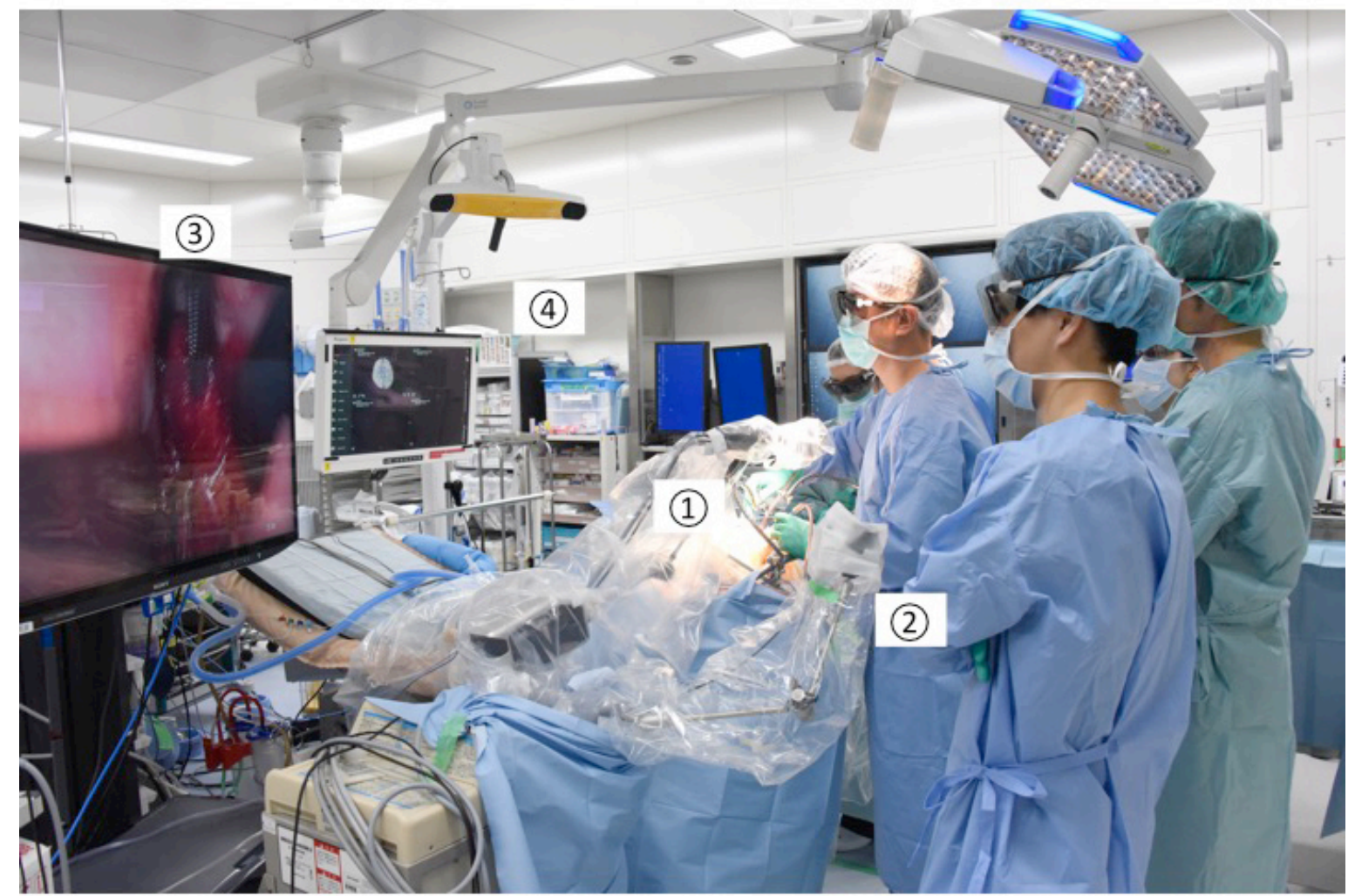

Fig. 1. Intraoperative setup for exoscopic corpus callosotomy. The VITOM 3D exoscope (1) is positioned cranial to the patient. (2) Controller of the camera and other systems. (3) The $4 \mathrm{~K}-3 \mathrm{D}$ surgical monitor. (4) Neuronavigation system.

VNS therapy did not change the frequency or intensity of spasms, which were considered to carry a risk of inducing severe traumatic injuries. His parents were thus dissatisfied with the results. Consequently, we planned CC as palliative therapy for the spasms.

\section{Surgical technique}

Under general anesthesia, the patient was positioned supine on the operating table with head fixed in a Mayfield head clamp. A neuronavigation system (iPlan Cranial software; Brainlab AG, Feldkirchen, Germany) was introduced to the surgical field. We made a small bicoronal skin incision around the bregma. With four burr holes, placed 3 $\mathrm{cm}$ anterior, $2 \mathrm{~cm}$ posterior, $3 \mathrm{~cm}$ to the right, and $3 \mathrm{~cm}$ to the left of the bregma, craniotomy was performed, then a wide U-shaped dural flap was made on the right side. We did not minimize the skin incision and craniotomy for use of the exoscope in case we needed to switch to using the microscope. Retractor blades were set over the cortex and falx, exposing the inferior falx and cingulate gyri. At this time, the $4 \mathrm{~K}-3 \mathrm{D}$ surgical monitor was placed caudal to the patient and in front of the operator. All medical staff wore surgical 3D glasses, and performed the steps while viewing the same images (Fig. 1).

Under the exoscope (EX VITOM 3D; Karl Storz, Tuttlingen, Germany), interhemispheric adhesions were judiciously divided. We identified the corpus callosum, then retractor blades were advanced. Pericallosal arteries were gently mobilized to develop a working space on the surface of the corpus callosum. The callosal division was initiated with two microsuction tubes at the level of the body of the corpus callosum. The callosal transection was performed in between the two leaves of the septum pellucidum at the midline cleft, and the inferior edge of the disconnection was the ependyma of the lateral ventricles $[10,12]$. As the dissection progressed anteriorly through the genu and rostrum to the level of the superior lamina terminalis, anterior cerebral arteries were visualized as they looped around the rostral aspect of the corpus callosum. As the dissection was advanced posteriorly, the retractors were repositioned. We finished this procedure on the anterior side of the isthmus, which was the anterior twothirds of the corpus callosum. Grossly confirming the narrow part of the corpus callosum, the position of the isthmus was reconfirmed using image guidance.

\section{Results}

\subsection{Clinical course}

The patient experienced transient right hemispatial neglect, lasting one week. No typical disconnection syndrome was seen, including transient disturbance of consciousness. Postoperative EEG revealed a decreased frequency of bilateral synchronous discharges. The frequency of seizures was reduced to approximately 2-3 times/week. Although he still 


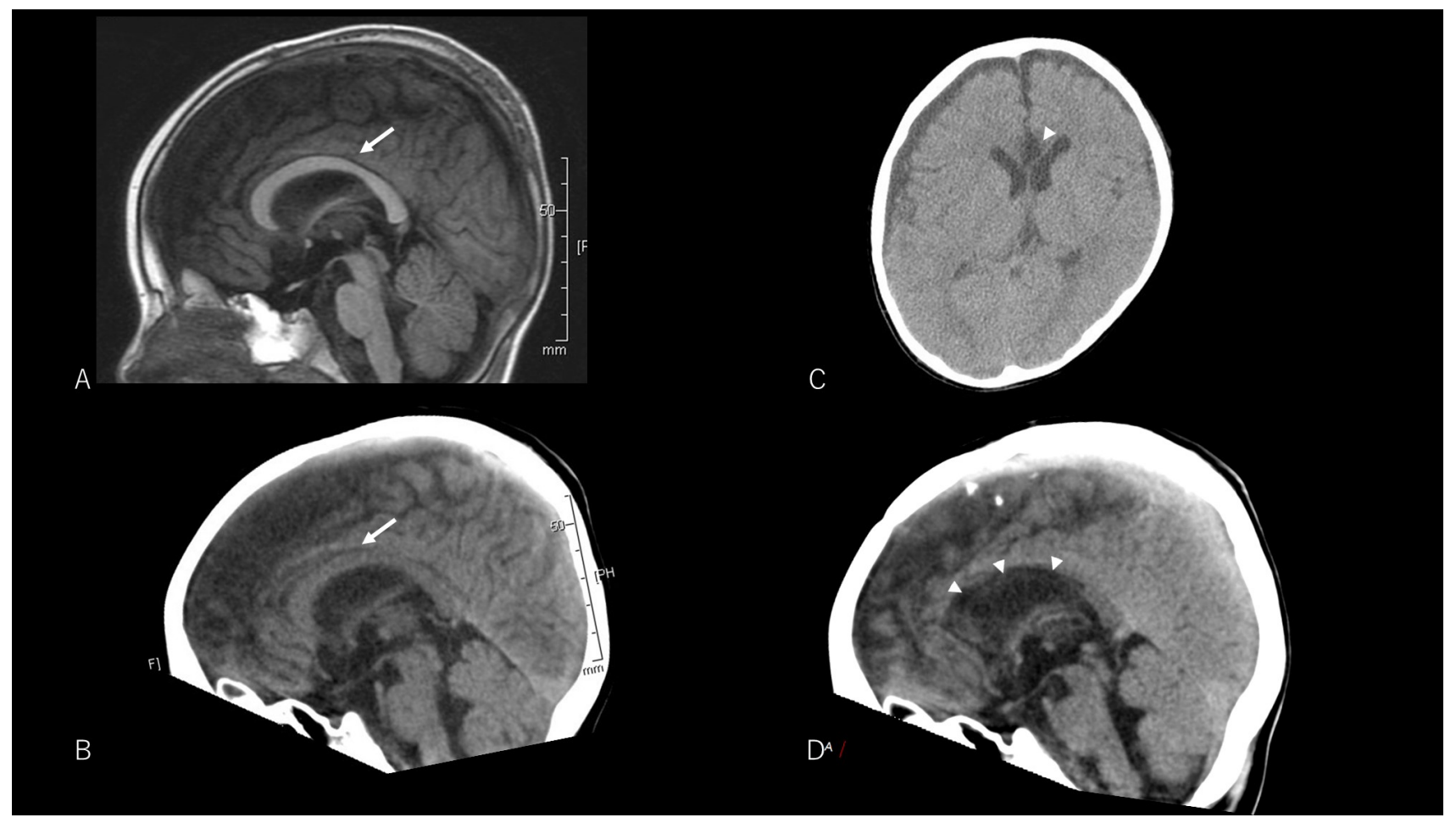

Fig. 2. Pre- and postoperative neuroimaging. Preoperative T1-weighted image (A) and computed tomography (CT) image (B) both show the corpus callosum (arrow). Postoperative CT image shows a low-density area at the anterior corpus callosum (arrowheads) indicating successful corpus callosotomy (C and D).

exhibited spasms, the intensity of seizures was reduced and no traumatic injury secondary to seizures was observed during the 9-month postoperative follow-up period. Postoperative computed tomography showed successful CC (Fig. 2).

\subsection{Operative time}

This case required $134 \mathrm{~min}$ for exoscopic CC. As a reference, we performed 20 microscopic callosotomies in our hospital between April 2017 and September 2020, and the mean required time was $158 \mathrm{~min}$ (median, $148 \mathrm{~min}$ ).

\subsection{Optical quality}

The visual quality of magnified images on the $4 \mathrm{~K}-3 \mathrm{D}$ monitor produced by the exoscope was uniformly perceived by all surgeons and medical staff. Stereoscopic vision was easily obtained. Moreover, we felt that the exoscope provided higher-quality magnification, compared to the operating microscope (Fig. 3). Luminosity of the exoscope light was high, providing a bright, broad surgical field. For epilepsy surgery that deals with normal-appearing white matter fibers, rather than brain tumors or vascular abnormalities, being able to adjust the luminosity intraoperatively is particularly important because white matter is easily blurred by halation effects. If the exoscope light was too bright, and the corpus callosum was blurred due to halation effects, we decreased the illumination. Under lower light conditions, we were able to confirm the anatomy without difficulty.



Fig. 3. View of the surgical field. Exoscopic corpus callosotomy shows a wider view than microscopic callosotomy.

\subsection{Surgeon impressions}

The primary operator (AF) did not feel any stress during the exoscopic surgery, but some headache and dizziness af- 
ter the procedure. He felt that the exoscopic CC was easier than microscopic callosotomy, because there were almost no restrictions on the visual axis. He therefore felt that he could obtain a wider field of view with exoscopic CC than with microscopic callosotomy. The first and second assistants in this surgery (TN and KT, respectively) felt that the exoscope provided help in training surgeons. Sharing the same view and quality of vision with surgeons allows clear communication and understanding of surgical nuances. In addition, when switching positions from the assistant to the primary operator, no barriers to the terms of surgical orientation are seen compared to microsurgical callosotomy.

\section{Discussion}

We report herein our first experience with exoscopic CC. Van Wagenen and Herren [13] first described CC in 1940 and Wilson et al. [14] reported CC using the operative microscope in 1982. Endoscopic CC was first described by Bahuleyan et al. [15] in cadaveric dissection in 2011. The current three-hand technique introduced by Chandra allows surgeons to perform endoscopic CC and manage hemorrhage in cases of vascular injury [10, 16]. Our experience introducing the exoscope to the neurosurgical field, as shown in the present case, suggests the exoscope may work well even in CC.

An exoscope not only provides magnification, lighting, and high-definition images comparable to the microscope, and helps to address the specific difficulties faced by the operators for structures deeper and further from the craniotomy, as described above, but also outperforms the microscope in some regards. First, because the exoscope is small and lightweight, it has excellent operability. Second, the exoscope allows the visual axis to be easily changed, as with the case when we examined the corpus callosum from anterior to posterior. The surgeon and assistant often have to remain in uncomfortable postures for some time when using the microscope, which may influence the level of concentration of the surgeons and the surgical outcomes. We felt that the exoscope largely solves this problem and allows maintenance of a comfortable posture during surgery. Third, the microscope has a heavier scope head and narrower visual field than the exoscope, and sometimes provides a frame-by-frame surgical field rather than an uninterrupted surgical field when changing the scope angle. However, since the exoscope provides easy manipulation and a wider visual field than the microscope, changes in the scope angle are smooth and uninterrupted, preventing disorientation of the surgeons.

Although 3D monitors allow stereoscopic vision, watching a $3 \mathrm{D}$ monitor for a prolonged time can lead to headache or dizziness, as experienced by the primary operator. In this regard, more experience and practice are needed.

The exoscope offers many advantages. As a new and evolving technology, improvements in next-generation devices are anticipated.

\section{Conclusions}

We report our first clinical experience with exoscopic CC. The exoscope appears to be a highly useful device in the field of epilepsy surgery.

\section{Abbreviations}

CC, corpus callosotomy; EEG, electroencephalography; $3 \mathrm{D}$, three-dimensional; VNS, vagus nerve stimulation.

\section{Author contributions}

All authors made substantial contributions to the conception, validation, design, acquisition of data, or analysis and interpretation of data, including methodology (AF), investigation $(\mathrm{KT}, \mathrm{AF}, \mathrm{CI})$, resources $(\mathrm{AF}, \mathrm{IC}, \mathrm{TN})$, data curation (KT, TN, CI, AF), writing the original draft (KT), writing, review, and editing $(\mathrm{AF})$, visualization $(\mathrm{TN})$, supervision $(\mathrm{AF}$, $\mathrm{CI})$, and project administration (AF).

\section{Ethics approval and consent to participate}

We confirm that all authors have read the Journal's position on issues involved in ethical publication and affirm that this report is consistent with those guidelines. We also confirm that this research received approval from the institutional review board. Submission of this case report was approved by the ethics review board at Seirei General Hospital, and written informed consent was obtained from the parents of the patient for the exoscopic CC procedure and publication of the case details.

\section{Acknowledgment}

We are grateful to the clinical engineering staff at Seirei Hamamatsu General Hospital.

\section{Funding}

This research received no external funding.

\section{Conflict of interest}

The authors declare no conflict of interest.

\section{References}

[1] Mamelak AN, Danielpour M, Black KL, Hagike M, Berci G. A high-definition exoscope system for neurosurgery and other microsurgical disciplines: preliminary report. Surgical Innovation. 2008; 15: 38-46.

[2] Carlucci C, Fasanella L, Ricci Maccarini A. Exolaryngoscopy: a new technique for laryngeal surgery. Acta Otorhinolaryngologica Italica. 2012; 32: 326-328.

[3] Marzano E, Piardi T, Soler L, Diana M, Mutter D, Marescaux $\mathrm{J}$, et al. Augmented reality-guided artery-first pancreaticoduodenectomy. Journal of Gastrointestinal Surgery. 2013; 17: 1980-1983.

[4] Ntourakis D, Memeo R, Soler L, Marescaux J, Mutter D, Pessaux P. Augmented Reality Guidance for the Resection of Missing Colorectal Liver Metastases: an Initial Experience. World Journal of Surgery. 2016; 40: 419-426.

[5] Pafitanis G, Hadjiandreou M, Alamri A, Uff C, Walsh D, Myers $\mathrm{S}$. The Exoscope versus operating microscope in microvascular surgery: a simulation non-inferiority trial. Archives of Plastic Surgery. 2020; 47: 242-249. 
[6] Mamelak AN, Nobuto T, Berci G. Initial clinical experience with a high-definition exoscope system for microneurosurgery. Neurosurgery. 2010; 67: 476-483.

[7] Oertel JM, Burkhardt BW. Vitom-3D for Exoscopic Neurosurgery: Initial Experience in Cranial and Spinal Procedures. World Neurosurgery. 2017; 105: 153-162.

[8] Nossek E, Schneider JR, Kwan K, Kulason KO, Du V, Chakraborty $\mathrm{S}$, et al. Technical Aspects and Operative Nuances Using a HighDefinition 3-Dimensional Exoscope for Cerebral Bypass Surgery. Operative Neurosurgery. 2019; 17: 157-163.

[9] Tubbs RS, Smyth MD, Salter G, Doughty K, Blount JP. Eyebrow incision with supraorbital trephination for endoscopic corpus callosotomy: a feasibility study. Child's Nervous System. 2004; 20: 188-191.

[10] Smyth MD, Vellimana AK, Asano E, Sood S. Corpus callosotomyOpen and endoscopic surgical techniques. Epilepsia. 2017; 58: 7379.

[11] Sufianov AA, Cossu G, Iakimov IA, Sufianov RA, Markin ES, Stefanov SZ, et al. Endoscopic Interhemispheric Disconnection for
Intractable Multifocal Epilepsy: Surgical Technique and Functional Neuroanatomy. Operative Neurosurgery. 2020; 18: 145157.

[12] Fountas K, Kapsalaki EZ. Epilepsy Surgery and Intrinsic Brain Tumor Surgery. 1. Springer: Cham. 2017.

[13] Van Wagenen WP, Herren RY. Surgical division of commissural pathways in the corpus callosum. Archives of Neurology \& Psychiatry. 1940; 44: 740-759.

[14] Wilson DH, Reeves AG, Gazzaniga MS. "Central" commissurotomy for intractable generalized epilepsy: series two. Neurology. 1982; 32: 687-687.

[15] Bahuleyan B, Vogel TW, Robinson S, Cohen AR. Endoscopic total corpus callosotomy: cadaveric demonstration of a new approach. Pediatric Neurosurgery. 2011; 47: 455-460.

[16] Chandra SP, Kurwale NS, Chibber SS, Banerji J, Dwivedi R, Garg A, et al. Endoscopic-Assisted (through a Mini Craniotomy) Corpus Callosotomy Combined with Anterior, Hippocampal, and Posterior Commissurotomy in Lennox-Gastaut Syndrome. Neurosurgery. 2016; 78: 743-751. 